\title{
FLEXIBLE STRUCTURE CONTROL SCHEME OF A UAVS FORMATION TO IMPROVE THE FORMATION STABILITY DURING MANEUVERS
}

\author{
Cezary KOWNACKI*, Leszek AMBROZIAK* \\ *Faculty of Mechanical Engineering, Department of Automatics and Robotics, Bialystok University of Technology, \\ ul. Wiejska45C, 15-351 Bialystok, Poland \\ c.kownacki@pb.edu.pl, l.ambroziak@pb.edu.pl
}

received 10 October 2016, revised 18 July 2017, accepted 7 August 2017

\begin{abstract}
One of the issues related to formation flights, which requires to be still discussed, is the stability of formation flight in turns, where the aerodynamic conditions can be substantially different for outer vehicles due to varying bank angles. Therefore, this paper proposes a decentralized control algorithm based on a leader as the reference point for followers, i.e. other UAVs and two flocking behaviors responsible for local position control, i.e. cohesion and repulsion. But opposite to other research in this area, the structure of the formation becomes flexible (structure is being reshaped and bent according to actual turn radius of the leader. During turns the structure is bent basing on concentred circles with different radiuses corresponding to relative locations of vehicles in the structure. Simultaneously, UAVs' airspeeds must be modified according to the length of turn radius to achieve the stability of the structure. The effectiveness of the algorithm is verified by the results of simulated flights of five UAVs.
\end{abstract}

Key words: Unmanned Aerial Vehicles, UAVs Formation, Rigid Formations, Flexible Formations

\section{INTRODUCTION}

The problem of UAVs formations flights has been intensively studied for many years in many research centers all over the world. Most of the research in this field is focused on three different approaches, i.e. formation flights based on a rigid virtual structure (Norman and Hugh, 2008; Ren and Beard, 2004; J. Shan and Liu, 2005; Cai et al., 2012; Seo et al., 2009; Askari et al., 2015) or a flexible virtual structure (Low and $\mathrm{Ng}, 2011$ ), swarms using biologically inspired flocking behaviors (Quintero et al., 2013; Kownacki and Ołdziej, 2015; 2016; Virágh et al., 2014) and relations based on the model of leader - follower (Xingping et al., 2003; Yun et. al., 2008; Ambroziak and Gosiewski, 2014). In the first approach, UAVs create a rigid structure, where relative distances between UAVs should be constant during a flight as much as it is possible (Norman and Hugh, 2008). Achieving this becomes a challenge because it requires not only precision control but also real-time motion synchronization. Therefore, in Low and $\mathrm{Ng}$, (2011) a model of flexible virtual structure is proposed, where relative distances can be slightly varied during turns. But proposed structure of control is centralized and it does not consider a collision risk inside the formation as it relies on local generation of reference trajectory for each UAV on basis of the reference trajectory of the leader. The second approach applies behaviors which are inspired by the flocking behaviors of birds. Also, in this case, local flight control depends on information sharing at least between the nearest neighbors of the vehicle (Kownacki and Ołdziej, 2015; 2016). Otherwise, the vehicle cannot determine its own behaviors, especially repulsion or cohesion. The last approach, based on the leader-follower relation, is relatively the simplest one, but as it was proved it requires control switching between position control and the course control to achieve the effective flight (Ambroziak and Gosiewski, 2014).

Despite some progress in the field of multi-UAV systems, a control algorithm, which would offer the effective flight control in real applications for fixed-wing UAVs, has not been thoroughly developed yet. This fact is especially related to the issue of the stability of the formation flight in turns, where each vehicle makes a turn under different aerodynamic conditions as the result of different speeds and different bank angles. Problems with finding an appropriate solution arise from the limitations of available technology and the nature of small fixed-wing UAVs being nonholonomic robots, whose high dynamics combined with small time constants make them sensitive to any external disturbances. Therefore, a position control in a formation requires a real-time processing of navigational data acquired from others UAVs, and it should involve especially a problem of synchronization of flight parameters and their actual errors (Norman and Hugh, 2008). In turn, this requires a lot of bytes to be transmitted smoothly through a wireless communication network inside the formation, what in most cases becomes another technological problem.

To avoid these issues, in contrast to the model in Low and $\mathrm{Ng}$, (2011), we propose a decentralized control algorithm based on a leader as the reference point for other UAVs and two flocking behaviors responsible for local position control, i.e. cohesion and repulsion. The flow of navigational data is organized on the model of cascade, in which transmission is initialized by the leader, whose data is sequentially forwarded by next follower in the structure. This simplifies the structure of wireless network inside the formation. But opposite to our previous research (Kownacki and Ołdziej, 2015; 2016), the structure of the formation becomes flexible (the structure is being reshaped and bent according to actual turn radius of the leader. During turns, the structure is bent basing on concentered circles with different radiuses corresponding to relative locations of vehicles in the structure. Simultaneous- 
ly, UAVs' airspeeds must be modified according to length of turn radius to achieve the stability of the structure.

\section{FORMATION FLIGHT WITH ALGORITHM OF FLEXIBLE STRUCTURE}

Regardless to the applied control algorithm, a typical formation flight assumes that relative distances between vehicles must be constant as much as possible. Therefore, UAVs formation usually uses structures, which define reference positions of each UAV in relation to a chosen reference point. The most convenient way, from the practical point of view, is to use a leader of a formation as the reference point to determine desired positions of other UAVs, which will play the role of followers. According to this, the leader must broadcast its actual position and orientation angles to allow the followers to calculate their reference positions in the structure, which become set-points for the local control of UAV. In the proposed approach, local low level control is based on two flocking behaviors, i.e. cohesion and repulsion (Kownacki and Ołdziej, 2015; 2016). The shape of the formation structure may be any as long as the distances between vehicles are exactly defined in a local coordinates frame associated with the formation. In the presented studies, a shape of reversed letter ' $V$ ' is used to create a simulated formation of five UAVs. This kind of structure will allow determining clearly noticeable differences between trajectories of the followers flying on opposite sides of the formation structure. A simulated structure of UAVs formation with local coordinate system for reference positions is presented on Fig. 1.

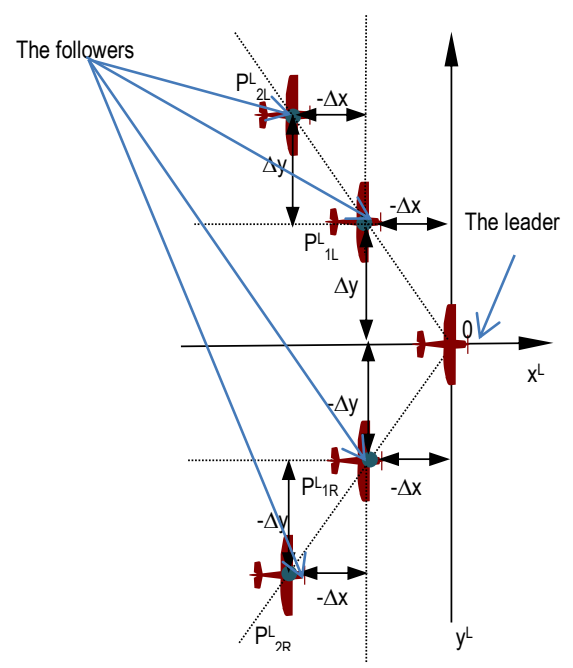

Fig. 1. A structure of the formation of five UAVs based on the shape of reversed letter ' $V$ '

According to the formation structure presented in Fig. 1, reference positions of UAVs can be determined by predefined points located in a local coordinate frame $L$, whose origin is placed at the gravity centre of the leader, and its axes are parallel to the axes of the leader's body. Therefore, coordinates of these points can be expressed by equation (1). In the equation, index $i$ in the subscripts refers to the order of UAVs placed behind and in reference to the leader, identically on its both sides. While index $j$ refers respectively to the left $(j=L)$ and to the right $(j=R)$ side of the leader.
$P_{i j}^{L}=\left[\begin{array}{c}x_{i j}^{L} \\ y_{i j}^{L} \\ z_{i j}^{L}\end{array}\right]=\left\{\begin{array}{c}{\left[\begin{array}{c}-i \cdot \Delta x \\ i \cdot \Delta \mathrm{y} \\ 0\end{array}\right] j=R} \\ {\left[\begin{array}{c}-i \cdot \Delta x \\ -i \cdot \Delta \mathrm{y} \\ 0\end{array}\right] j=L}\end{array}\right.$

where: $i$ - the order of UAVs in chain on the left side $(j=L)$ or on the right side $(j=R)$ in reference to the leader, $\Delta x, \Delta y-$ spacings between UAVs, respectively in $x^{L}$ and $y^{L}$ axis.

If the formation structure is rigid, the coordinates of those points $P_{i j}^{L}$ in the global frame $G$, which is related to local horizon and the north, will be determined by a transformation, which combines a shift of coordinates by the leader's coordinates given in the frame $G$ and a rotation around the leader's gravity centre about its orientation angles i.e. roll, pitch and heading. The transformation can be defined by a following equation:

$P_{i j}^{G}=P_{L D}^{G}+D_{G}^{L}\left(\phi_{L D}, \theta_{L D}, \psi_{L D}\right) \cdot P_{i j}^{L}$

where: $D_{G}^{L}$ - a rotation matrix defining elementary rotations between the formation frame $L$ and the inertial frame $G, P_{L D}^{G}-$ the position of the leader in the inertial frame $G, P_{i j}^{G}$ - the reference position of the $i$-th UAV flying on the right $(j=R)$ or left side $(j=L)$ of the leader given in the inertial frame $G, P_{i j}^{L}$ - the reference position of the $\mathrm{i}$-th UAV on the right $(j=R)$ or left side $(j=L)$ of the leader given in the local frame $L, \psi_{L D}$ - a heading angle of the leader, $\phi_{L D}-$ a roll angle of the leader, $\theta_{L D}-$ a pitch angle of the leader.

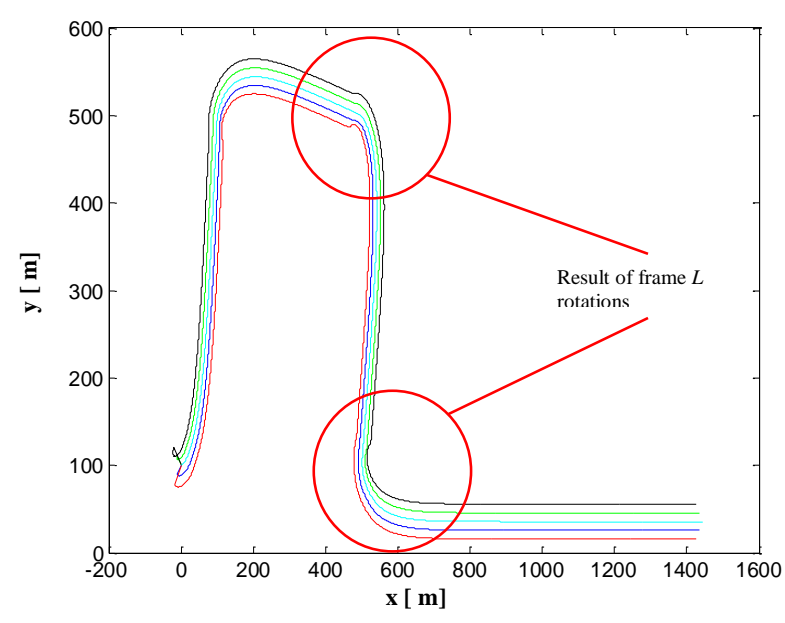

Fig. 2. Ground projections of trajectories of reference UAVs positions for a formation flight based on a rigid structure

Unfortunately, the approach based on the rigid structure can result in deformations of reference trajectories, which are evolutions of points $P_{i j}^{G}$ in time, especially while the formation makes a turn maneuver. Rotations of the local frame $L$ around the leader's gravity centre make turn radiuses of the followers greater than it is in the case of the leader and finally the formation structure is disturbed as it is shown in Fig. 2.

The deformations of UAVs trajectories in Fig. 2 are a strong argument against the use of the approach of the rigid structure. Therefore, a new approach, which will use a flexible structure should be proposed. In the approach of the flexible structure, the formation structure will be constantly modified according to actual turn radius of the leader. This means also that the reference positions of the followers will be modified in relation to the refer- 
ence point, and relative distances between UAVs will not be constant. In the flexible structure, reference positions of the followers during turns can be defined by finding points of intersections between concentric circles, whose radiuses are determined by a sum of turn radius of the leader $(R)$ and coordinates of UAVs reference positions in $y$-axis of the frame $L(\Delta y)$, and lines passing through the center of these circles. The lines will create arcs on the trajectory of the leader, which start in the position of the leader, and whose widths are equal to $i \cdot \Delta x$, coordinate of reference position in $x$-axis of frame $L$ for the i-th UAV. The idea of the flexible structure of a UAVs formation is presented in Fig. 3.

The main rule of the flexible structure in Fig. 3 can be defined briefly as the change of expression of reference positions of UAVs in the local frame $L$ from Cartesian coordinates to polar coordinates only when the leader's roll angle is different from zero. In both coordinates systems, relative coordinates of UAVs positions in reference to the leader, i.e. positive and negative multiplications of $\Delta x$ and $\Delta y$ (eq. 1), remain the same, but in the case of polar coordinates system, they are given as widths of arcs and differences in lengths of turn radiuses. Moreover, the origin of polar coordinates system is located at the center of a circle being a part of the leader's trajectory. To determine reference positions of UAVs in the frame $G$, it is necessary to identify a Cartesian representation of relative distances $\Delta x$ and $\Delta y$ expressed as polar coordinates. Let's start with a definition of concentric circles in the frame $L$, which is as follows:

$\left\{\begin{array}{c}x_{i j}^{2}+\left(y_{i j}-R\right)^{2}=\left(R-y_{i j}^{L}\right)^{2}, \phi<0, \\ x_{i j}^{2}+\left(y_{i j}+R\right)^{2}=\left(-R-y_{i j}^{L}\right)^{2}, \phi \geq 0 .\end{array}\right.$

where: $R$ - turn radius of the leader, $x_{i j}, y_{i j}$ - coordinates of points forming trajectory of the $\mathrm{i}$-th UAV flying on the right $(j=R)$ or on the left side $(j=L)$ of the leader, given in the frame $L, y_{i j}^{L}$-coordinate of the reference position in $y$-axis of the frame $L$ for the i-th $U A V$, which is flying on the right $(j=R)$ or on the left side $(j=L)$ of the leader, $\phi$-actual roll angle of the leader.

In the next step, equations of lines should be defined. These lines intersect the leader's trajectory. Intersection points together with y-axis specify arcs widths equal to $x_{i j}^{L}$-coordinates of the reference positions of UAVs in x-axis of the $L$ frame. Angles between those lines and $y$-axis of the $L$ frame are given by equation (4).

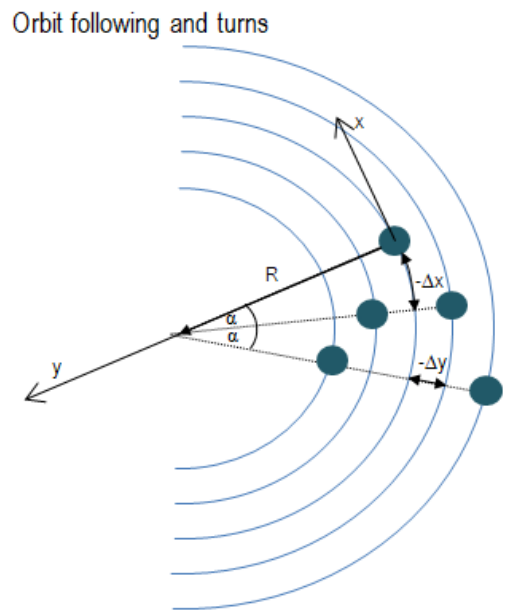

$\alpha_{i j}=\frac{\left|x_{i j}^{L}\right|}{|R|}$.

where: $R$ - turn radius of the leader, $x_{i j}^{L}$ - coordinate of reference position in $x$-axis of the frame $L$ for the i-th UAV flying on the right $(j=R)$ or on the left $(j=L)$ side of the leader.

Therefore, equations of those lines can be formulated as follows:

$\left\{\begin{array}{c}y_{i j}-R=x_{i j} \cdot \operatorname{tg}\left(\frac{\pi}{2}-\alpha_{i j}\right), \phi<0, \\ y_{i j}+R=-x_{i j} \cdot \operatorname{tg}\left(\frac{\pi}{2}-\alpha_{i j}\right), \phi \geq 0 .\end{array}\right.$

If we take right sides of equations (5) and put them respectively into corresponding expressions on the left sides of equations (3), we will obtain $x_{i j}^{L F}$ - coordinate of reference position of UAV in $x$-axis of the frame $L$. Because the followers are always placed behind the leader, i.e. on the left side of $y$-axis of the frame $L$, the sign in the front of square root is negative.

If we take right sides of equations (5) and put them respectively into corresponding expressions on the left sides of equations (3), we will obtain $x_{i j}^{L F}$ - coordinate of reference position of UAV in $x$-axis of the frame $L$. Because the followers are always placed behind the leader, i.e. on the left side of $y$-axis of the frame $L$, the sign in the front of square root is negative.

$\left\{\begin{array}{l}x_{i j}^{L F}=-\sqrt{\frac{\left(R-y_{i j}^{L}\right)^{2}}{1+\operatorname{tg}^{2}\left(\frac{\pi}{2}-\alpha_{i j}\right)}}, \phi<0, \\ x_{i j}^{L F}=-\sqrt{\frac{\left(-R-y_{i j}^{L}\right)^{2}}{1+\operatorname{tg}^{2}\left(\frac{\pi}{2}-\alpha_{i j}\right)}}, \phi \geq 0 .\end{array}\right.$

To determine coordinates of UAVs reference positions in $y$ axis of the frame $L$, right sides of equations (6) should be substituted in the place of $x_{i j}$ in equations (5). The equation for coordinate $y_{i j}^{L F}$, is given as follows:

$$
\left\{\begin{array}{c}
y_{i j}^{L F}=-\operatorname{tg}\left(\frac{\pi}{2}-\alpha_{i j}\right) \cdot \sqrt{\frac{\left(R-y_{i j}^{L}\right)^{2}}{1+\operatorname{tg}^{2}\left(\frac{\pi}{2}-\alpha_{i j}\right)}}+R, \phi<0, \\
y_{i j}^{L F}=\operatorname{tg}\left(\frac{\pi}{2}-\alpha_{i j}\right) \cdot \sqrt{\frac{\left(-R-y_{i j}^{L}\right)^{2}}{1+\operatorname{tg}^{2}\left(\frac{\pi}{2}-\alpha_{i j}\right)}}-R, \phi \geq 0 .
\end{array}\right.
$$

\section{Straight line flight}

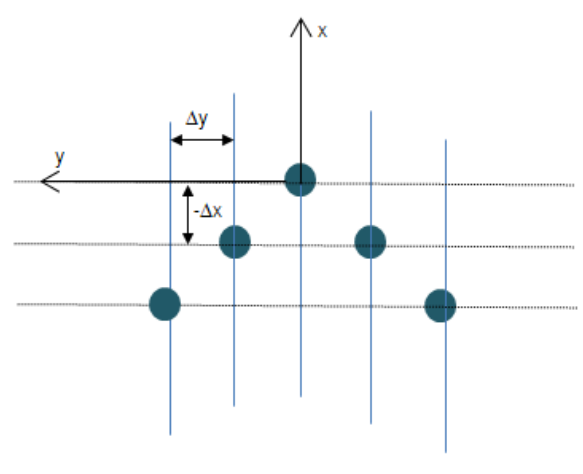

Fig. 3. The idea of the flexible structure of a UAVs formation. On the left side, there is the formation structure, which is modified according to the leader's turn radius $R$, and on the right side, there is the initial structure for a straight line flight 
Both equations (6) and (7) define together coordinates of reference positions of UAVs in the flexible structure in the frame $L$, in situation when the formation makes a turn or starts an orbitfollowing flight scheme. Coordinates in z-axis remain constant and they are equal to zero. To implement local controls based on the rules of birds flocking, each UAV must know its reference position given in the global frame $G$. But in contrast to the rigid structure approach, reference positions in the local frame $L$ should be rotated around the leader only about its heading angle. Hence, the transformation from the frame $L$ to the frame $G$ is given by equation (8), which splits the problem into separate cases, i.e. when an absolute value of the leader's roll angle is below and above value of $\boldsymbol{\phi}_{m}$, at which formation control switches to the flexible structure mode.

$$
P_{i j}^{G}=\left\{\begin{array}{c}
P_{L D}^{G}+D_{G}^{L}\left(\psi_{L D}\right) \cdot\left[\begin{array}{c}
x_{i j}^{L F} \\
y_{i j}^{L F} \\
0
\end{array}\right]|\phi|>\phi_{m}, \\
P_{L D}^{G}+D_{G}^{L}\left(\psi_{L D}\right) \cdot\left[\begin{array}{c}
x_{i j}^{L} \\
y_{i j}^{L} \\
0
\end{array}\right]|\phi|<\phi_{m} .
\end{array}\right.
$$

where: $D_{g}^{L}$ - rotation matrix defining a rotation between the frame $L$ and the inertial frame $G, P_{L D}^{G}$ - the position of the leader in the inertial frame $G, P_{i j}^{G}$ - the reference position for the $i$-th UAV, flying on the right $(j=R)$ or left side $(j=L)$ of the leader, given in the inertial frame $G, x_{i j}^{L F}, y_{i j}^{L F}$ - coordinates of the reference position of the i-th UAV flying on the right $(j=R)$ or left side $(j=L)$ of the leader, given in the local frame $L, \psi_{L D}$ - a heading angle of the leader, $\phi_{m}$ - the roll angle of the leader at which the formation control switches to the flexible structure mode.

Reference positions $P_{i j}^{G}$ in the frame $G$ are used to calculate local controls of UAV, whose main purpose is to minimize errors of position tracking. This means that the algorithm of the flexible structure becomes a high level of control, providing coordinates of reference positions as input for a middle level of controls i.e. a control of position tracking. In turn, the control of position tracking together with a necessary control of collision avoidance between UAVs generates set-points for a low-level controls which manages deflections of control surfaces of UAV. In this way, the overall control of UAV is organized in a form of three-stage cascade control, which is presented in Fig. 4.

As the base for both controls of position tracking and collision avoidance, flocking behaviors of birds, in particular, behaviors of cohesion and repulsion can be successfully applied, what was proved in the previous research ((Kownacki C. and Ołdziej D., 2015; Kownacki C. and Ołdziej D., 2016)). However here, the meaning of cohesion and repulsion behaviors should be adapted to the approach of formation flights. Therefore, the behavior of cohesion is used to move UAV towards its reference position instead of a gravity centre of a flock, and airspeed of UAV should be proportional to tracking errors or calculated with PID terms with a dead zone applied around the reference position. In turn, the behavior of repulsion secures UAV against collisions with another $U A V$, which is a preceding in the structure. The idea of applying behaviors of cohesion and repulsion in a formation flight is shown in Fig. 5.

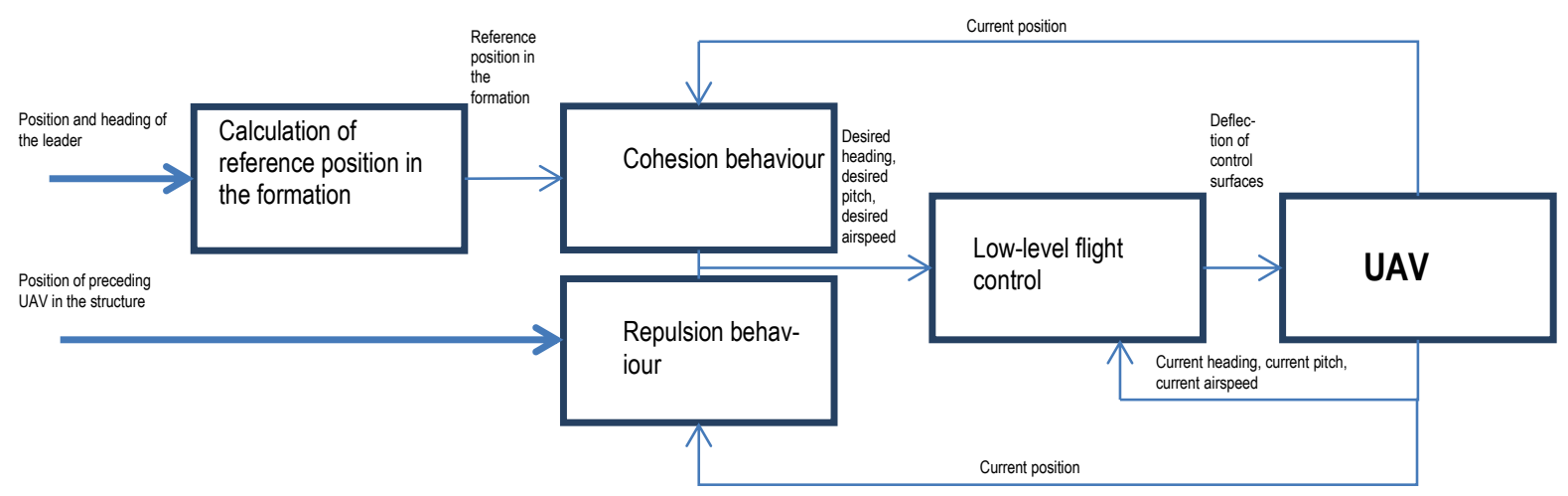

Fig. 4. The structure of the local control of UAV, which is organized in the form of tree-stage cascade control: the first stage - the algorithm of flexible structure, the second stage - flocking behaviors (cohesion and repulsion), the third stage - low level control of flight parameters, roll, pitch, heading and airspeed
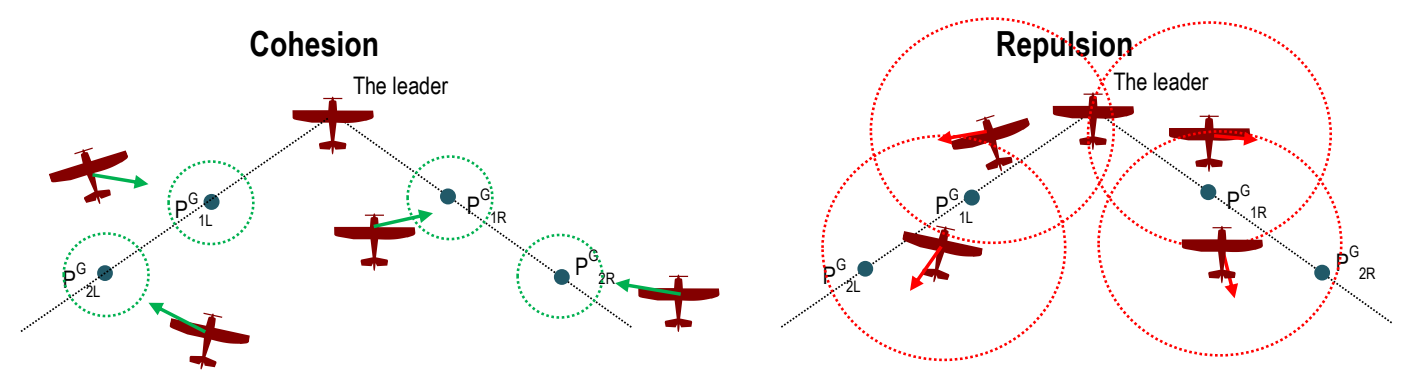

Fig. 5. The idea of using behaviors of cohesion and repulsion in the flight of formation based on the flexible structure. Green dotted circles represent dead zones around reference position, where there is no cohesion and airspeed is the same as the leader's, Red dotted circles represents zones of repulsion around each UAV with except of the leader. 
As it was mentioned, cohesion behavior moves UAV towards assigned reference position in the formation structure with an airspeed, which is proportional to a distance between an actual position of UAV and its reference position, and this distance has a meaning of a tracking error. Cohesion behavior is acting only when this tracking error is greater than specified maximal distance, which is a radius $D_{c}$ of dead zones around reference positions (green dotted circles in Fig. 5). Dead zones are required because they prevent from the instability of flight when the tracking error is nearby zero. Then even a small change of UAV position in relation to the assigned reference position can produce a rapid step change in control signals generated by cohesion behavior. Therefore, UAV should track its reference position keeping a specified distance and inside the dead zone its airspeed should be the same as the leader's. A direction of flight towards reference position determined by cohesion behavior is expressed as a vector defined by the equation below.

$\overrightarrow{K^{C}{ }_{l \jmath}}=\frac{1}{\left|\overrightarrow{A P_{l \jmath}^{G}}\right|} \cdot \overrightarrow{A P_{l \jmath}^{G}} \quad \overrightarrow{A P_{l \jmath}^{G}}=\overrightarrow{P_{l \jmath}^{G}}-\overrightarrow{P_{U A V_{l \jmath}}^{G}}$.

where: $\overrightarrow{A P_{l j}^{G}}$ - a vector which is defined as a difference between the coordinates $\left(\overrightarrow{P_{U A V_{l J}}^{G}}\right)$ of the i-th UAV flying on the right $(j=R)$ or left side $(j=L)$ of the leader, given in the inertial frame $G$ and the coordinates $\overrightarrow{P_{l j}}$ of assigned reference position in the virtual structure, $\left|\overrightarrow{A P_{l \jmath}^{G}}\right|$ - is a distance between the i-th UAV and the reference position in the structure. This is also position tracking error.

As it was mentioned, the airspeed of UAV should be proportionally adjusted to the tracking error and it should be constant or at least reduced to the half of the leader's speed when the tracking error is smaller than the radius $D_{c}$. Reducing the airspeeds of the followers allows decreasing their turn radiuses in reference to the leader's. Hence, a relation between airspeed and the tracking error is defined by equation (10):

$S_{i j}^{d}=$

$\left\{\begin{array}{c}K_{p} \cdot \cos (\phi) \cdot\left[\left|\overrightarrow{A P_{l j}^{G}}\right|+K_{I} \cdot \int_{0}^{t}\left|\overrightarrow{A P_{l j}^{G}}\right| d \tau+K_{D} \cdot \frac{d\left|\overrightarrow{A P_{l \jmath}^{G}}\right|}{d t}\right],\left|\overrightarrow{A P_{l j}^{G}}\right| \geq D_{c^{\prime}}{ }^{\prime}(10) \\ \beta \cdot S_{i}, \quad\left|\overrightarrow{A P_{l \jmath}^{G}}\right|<D_{c} .\end{array}\right.$

where: $K_{P}, K_{D}, K_{l}$ - gains of PID terms, $\left|\overrightarrow{A P_{l J}^{G}}\right|$ - the distance between the i-th UAV and its assigned reference position in the structure (the tracking error), $S_{i j}^{d}$ - the desired airspeed of the i-th UAV, $S_{i}$ - the leader's airspeed, $D_{c}-$ a radius of dead zones around the nodes in the virtual structure, $\phi$ - the leader's current roll angle, $\beta$-scaling factor to reduce speed when tracking error is lower than $D_{c}$.

The role of repulsion behavior is to secure the formation from collisions between UAVs. It repulses a UAV from another UAV which is a preceding in reference to the leader's position, only when distance between both UAVs is smaller than a safe distance $D_{R}$. Therefore, each UAV must know only the position of its precedor. This simplifies information sharing in the formation. A vector, which represents the direction of repulsion is as follows:

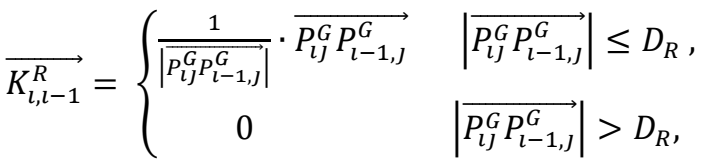

$\overrightarrow{P_{l j}^{G} P_{l-1, j}^{G}}=\overrightarrow{P_{l j}^{G}}-\overrightarrow{P_{l-1, \jmath}^{G}}$. where: $\overrightarrow{P_{l J}}, \overrightarrow{P_{l-1, j}^{G}}-$ positions respectively of the i-th and the (i-1)th vehicle flying on the left $(j=L)$ or the right $(j=R)$ side of the leader, $\left|\overrightarrow{P_{l j}^{G} P_{l-1, J}^{G}}\right|$ - a distance between UAVs, $D_{R}$ - the minimum permissible distance between two UAVs.

The current direction of UAV flight will be dependent on a combination of all behaviours - cohesion and repulsion, which is simply defined as a sum of vectors $\overrightarrow{K_{l, l-1}^{R}}$ and $\overrightarrow{K^{C}{ }_{l \jmath}}$. Vector $\overrightarrow{K_{l \jmath}}$, being a sum of vectors $\overrightarrow{K_{l, l-1}^{R}}$ and $\overrightarrow{K^{C}{ }_{l J}}$, is used to calculate setpoint for low-level flight control, i.e. desired pitch and desired heading.

$\overrightarrow{K_{l \jmath}}=\overrightarrow{K_{l, l-1}^{R}}+\overrightarrow{K^{C}{ }_{l \jmath}}$.

These set-points are defined by following equations.

$\overrightarrow{K_{l \jmath}}=\left[\begin{array}{l}x_{i j} \\ y_{i j} \\ z_{i j}\end{array}\right]$

$\Psi_{i j}^{d}=\operatorname{atan} 2\left(\frac{x_{i j}}{y_{i j}}\right)$,

$\Theta_{i j}^{d}=\operatorname{atan} 2\left(\frac{z_{j}}{\sqrt{x_{i j}^{2}+y_{i j}^{2}}}\right)$.

where: $\overrightarrow{K_{l j}}-$ a vector which defines the resultant direction of flight, $\Psi_{i j}^{d}$ - the desired heading angle, $\Theta_{i j}^{d}$ - the desired pitch angle.

The control vector, which is the input for low-level of flight control, can be finally defined as:

$\overrightarrow{u_{\imath \jmath}}=\left[\begin{array}{c}\Psi_{i j}^{d} \\ \Theta_{i j}^{d} \\ S_{i j}^{d}\end{array}\right]$.

To show the differences between the presented approach of flexible structure and virtual rigid structure a series of simulations were made in Matlab - Simulink. In the simulations, a formation of five UAVs flew through different sequences of three waypoints, each time it made at least one turn of about 90 degrees. Results of flights simulation making a comparison between rigid and flexible structure approaches are presented in next section.

\section{RESULTS}

To identify the main differences in both approaches to formation structures, the same sequence of waypoints and the same flight parameters were used to simulate flights, once based on the rigid structure algorithm and once on the proposed algorithm of the flexible structure. Making a comparison between trajectories of reference positions and UAVs for both approaches allows assessing how much the flexible structure improves the parallelism of UAVs trajectories, what is the main aim of the research. In simulation PD regulators are used to control airspeeds (eq. 10) and actual headings (low-level control) of the followers. Following parameters were also applied: the leader's airspeed $10 \mathrm{~m} / \mathrm{s}$, range of repulsion $D_{R}=1$ meter, the radius of dead-zones around reference positions in the structure $D_{C}=5$ meters, maximum roll angle for each UAV about $30^{\circ}$, initial 
headings $90^{\circ}$, initial positions of UAVs are placed in accordance to location in the structure of the formation. Fig. 6 presents trajectories of UAVs and trajectories of their reference positions for the case of the flexible structure approach on the left and for the case of the rigid structure approach on the right. In Fig. 7, it can be noticed that in the case of rigid structure, headings of outer UAVs in the structure differ from others, i.e. they oscillate in moments when they are flying on the inner sides of turns. But those differences do not correlate with the shape of reference trajectories what reflects in the fact that trajectories of the UAVs turn in opposite direction. Therefore, the most probable reason for the deformations in trajectories of the formation based on the rigid structure approach are overshoots in the roll angle control, which occur at the time of rapid changes of reference positions. And this happens only in the case of rigid structure, what can be proved by plots of roll angles in Fig. 8 and plots of reference heading in Fig. 9.
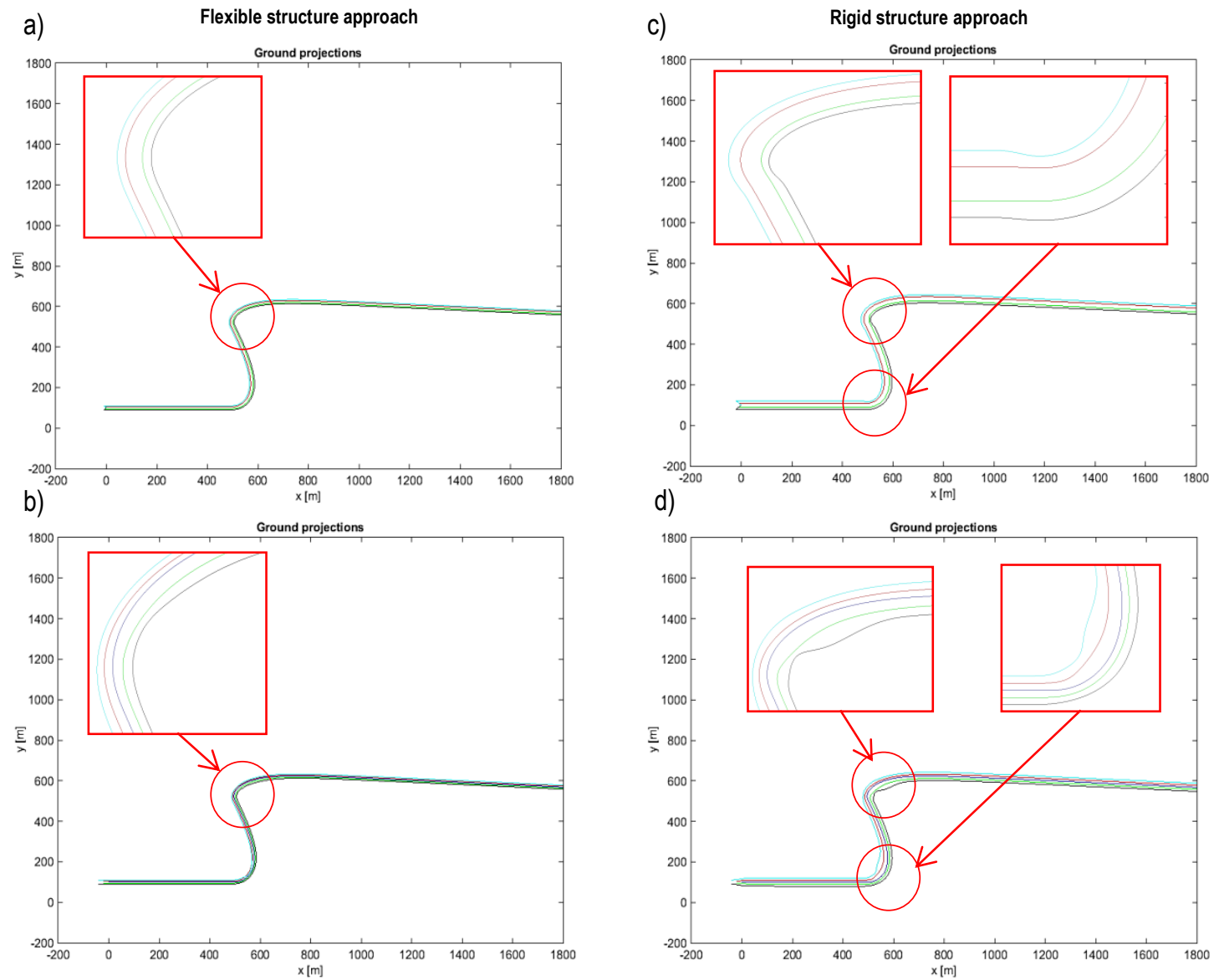

Fig. 6. Trajectories of reference positions and UAVs, respectively for the flexible structure approach: (a) - trajectories of reference positions, (b) - trajectories of UAVs, and the rigid structure approach: (c) - trajectories of reference positions, and (d) - trajectories of UAVs

a)

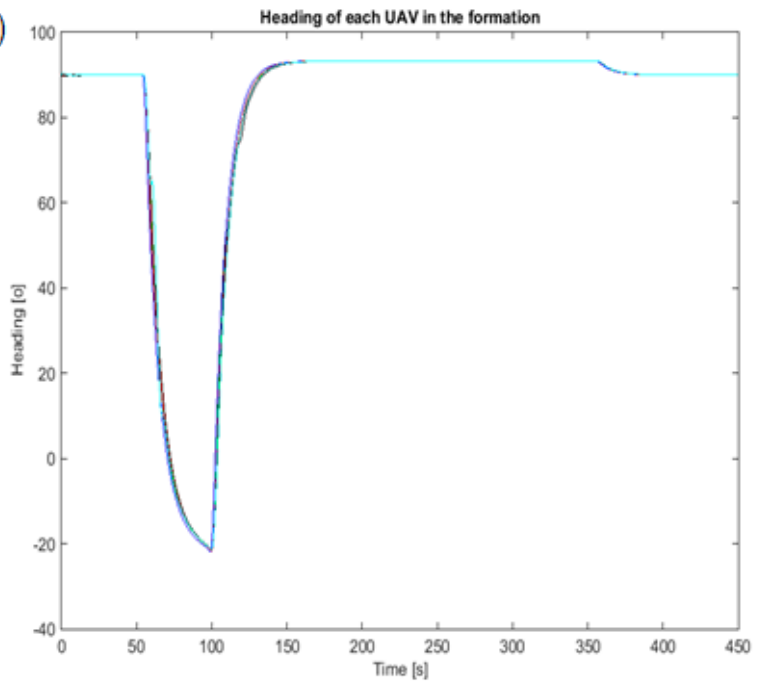

b)

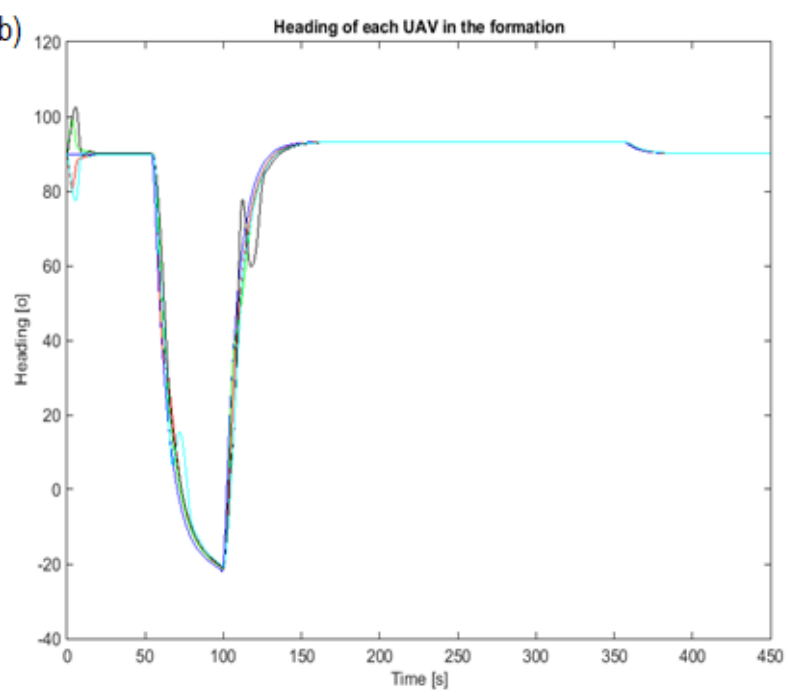

Fig. 7. Headings of UAVs, respectively for the flexible structure approach (a) and the rigid structure approach (b) 
Flexible Structure Control Scheme of a UAVs Formation to Improve The Formation Stability During Maneuvers
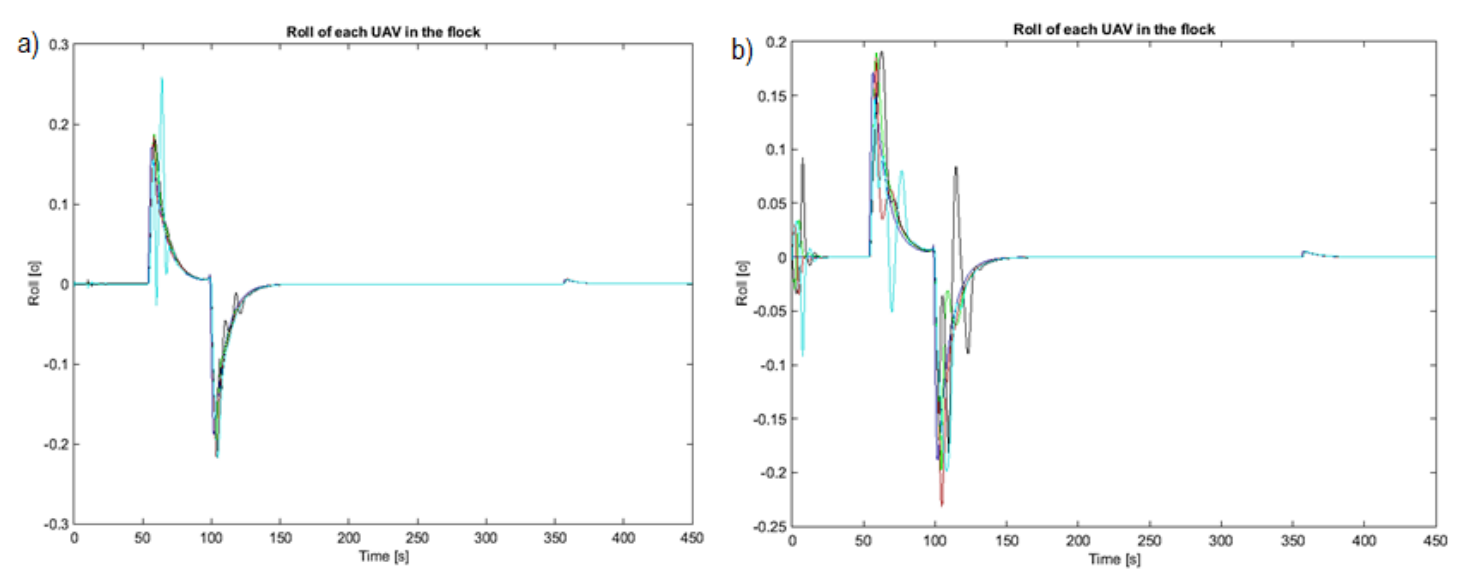

Fig. 8. Roll angles of each UAV in the formation, respectively for the flexible structure approach (a) and the rigid structure approach (b)
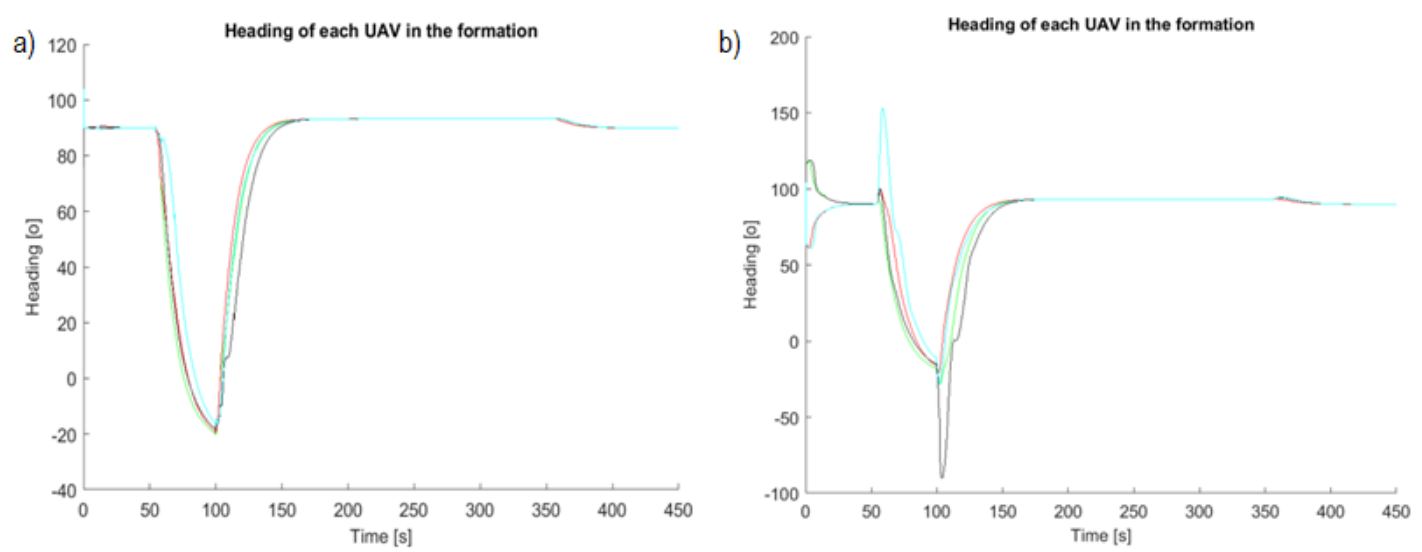

Fig. 9. Reference heading angles of followers in the formation, respectively for the flexible structure approach (a) and the rigid structure approach (b)

a)

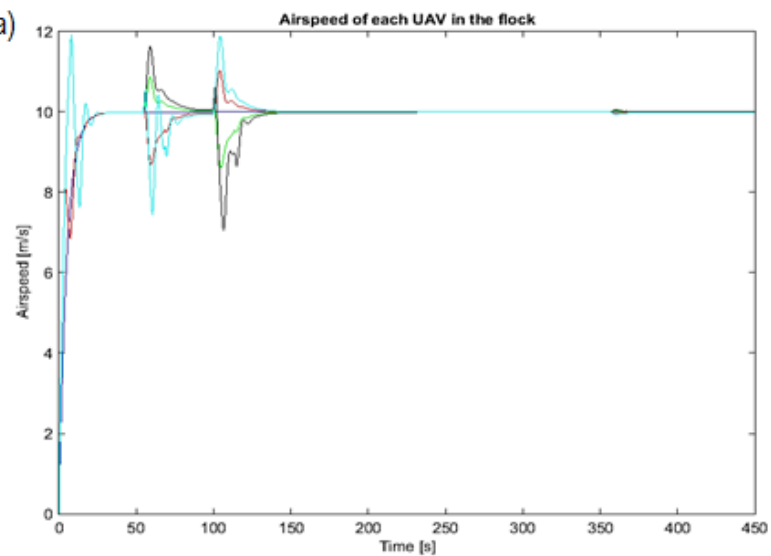

b)

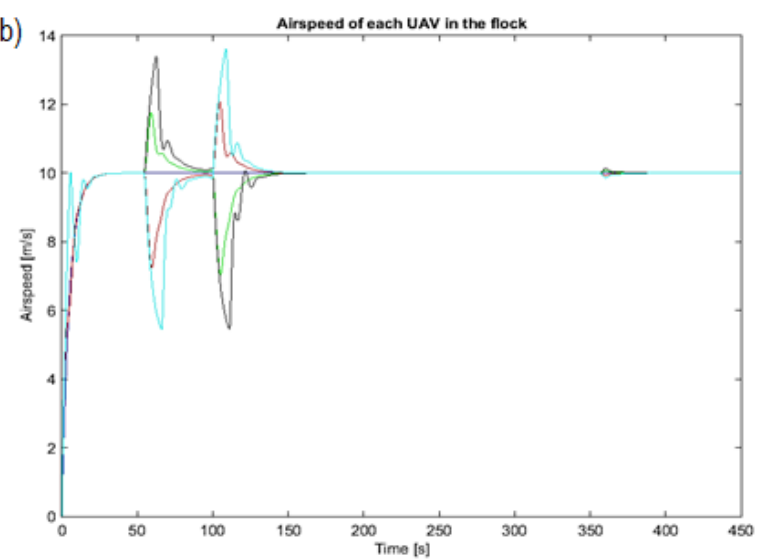

Fig. 10. Airspeeds of each UAV in the formation, respectively for the flexible structure approach (a) and the rigid structure approach (b)

a)

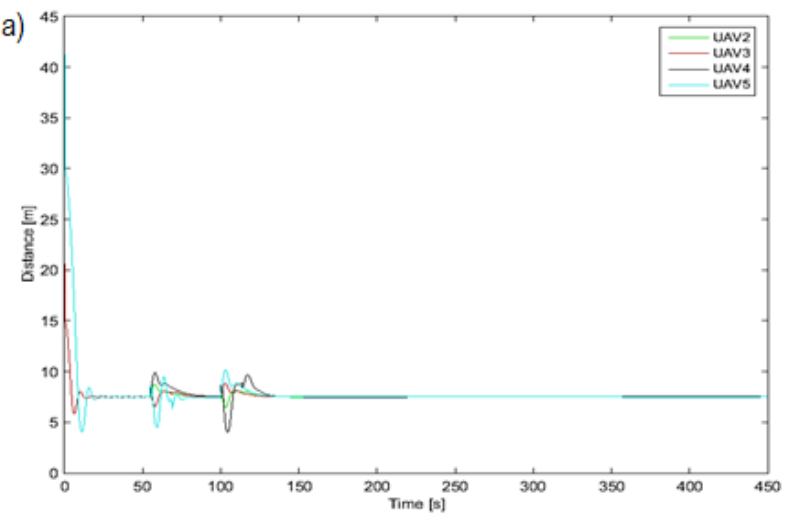

b)

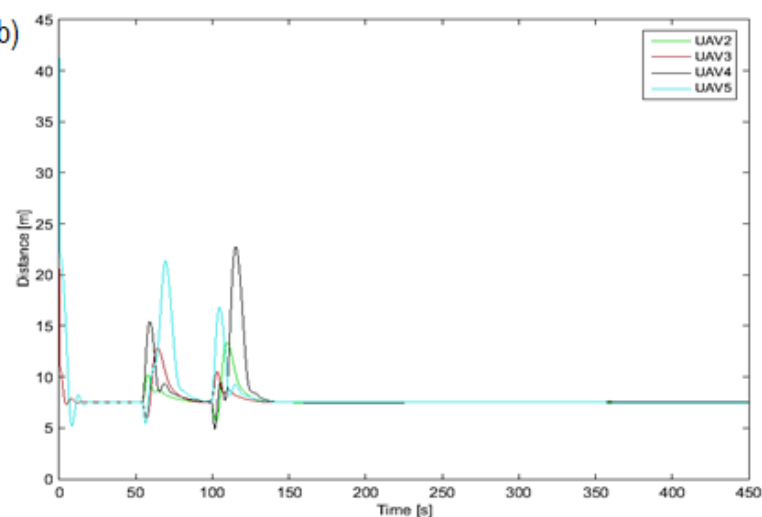

Fig. 11. Tracking error of each UAV in the formation, respectively for the flexible structure approach (a) and the rigid structure approach (b) 
Differences between plots of reference headings in Fig. 9 explain higher oscillations of roll angles in the case of the rigid structure approach, which next destabilize the structure of the formation. Therefore, trajectories of UAVs flying on the inner side of turn cease to be parallel to others. Applying the flexible structure instead of the rigid structure decreases these oscillations as the result of generating smoother reference trajectories for the followers. This situation is also confirmed by the position tracking error and airspeeds signals presented in Figs. 10 and 11 . Tracking errors are significantly lower during formation flight with flexible structure what also impacts on the control of airspeed as a function of them. Airspeed increases with the growth of tracking error and decreases if it is getting smaller.

\section{CONCLUSIONS}

The main purpose of the research on algorithms of formation flight designed to UAVs is achieving collective, synchronized and autonomous flight of several UAVs like it could be done by pilots. This still is difficult due to applied technology, which limits possibilities of real-time synchronization between UAVs. Therefore, the most of research is focused on approaches of leader-follower or virtual rigid structure, where the positions of UAVs are related to the leader's position by using predefined geometrical relations. This simplifies the exchange of navigation data between UAVs in the formation, but achieving constant geometrical relations, like it is in rigid structures. becomes more difficult in the case of nonholonomic robots, to which fixed-wing UAVs belong. Applying rigid structures to non-holonomic robots can result in structure rotations which deform trajectories of reference positions causing overshoots in angles of roll and heading.

In the research, the proposition of the new algorithm, which applies a flexible structure to organize formation flights is discussed. The main difference between the proposed algorithm and the approach based on virtual rigid structure is reshaping of the structure in accordance with the turn radius of the leader. Simulations results present that applying the flexible structure approach allows minimizing the impact of structure rotations when the leader changes its heading. Thus, coordinates of reference position do not change rapidly in relation to the UAV, what minimizes heading angle error and reduces oscillations of roll angle. In turn, a stable flight results in better parallelism of trajectories. Applied behaviors of cohesion and repulsion, together with dedicated airspeed control are enough to minimize positions tracking errors effectively. However, tracking errors cannot be smaller than the radius of the dead zone required to stabilize the flight when even a small position displacement can result in a step change of heading error. This issue can be eliminated by heading synchronization what will be the next step of the research.

\section{REFERENCES}

1. Ambroziak L., Gosiewski Z. (2014), Two stage switching control for autonomous formation flight of Unmanned Aerial Vehicles, Aerospace Science and Technology, 46, 221-226.

2. Askari A.; Mortazavi M.; Talebi H.A. (2015), UAV Formation Control via Virtual Structure Approach, Journal of Aerospace Engineering, 28(1), Article number: 04014047 (online).

3. Cai D., Sun J., Wu S. (2012), UAVs Formation Flight Control Based on Behavior and Virtual Structure, Communications in Computer and Information Science, 325, 429-438.
4. Kownacki C., Ołdziej D. (2015), Flocking Algorithm for Fixed-Wing Unmanned Aerial Vehicles, Advances in Aerospace Guidance, Navigation and Control, Springer, 415-431.

Kownacki C., Ołdziej D. (2016), Fixed-wing UAVs Flock Control through Cohesion and Repulsion Behaviours Combined with a Leadership, International Journal of Advanced Robotic Systems, Article number 36 (online),

5. Low Ch. B., Ng Q.S. (2011), A flexible virtual structure formation keeping control for fixed-wing UAVs, 9th IEEE International Conference on Control and Automation, 19-21 Decemeber, Santiago, 621-626.

6. Norman H. M. Li, Hugh H.T. Liu (2008), Formation UAV Flight Control using Virtual Structure and Motion Synchronization, American Control Conference, June 11-13, Seattle, USA, 1782-1787.

7. Quintero S.A.P.,, Collins G.E., Hespanha J.P. (2013), Flocking with Fixed-Wing UAVs for Distributed Sensing: A Stochastic Optimal Control Approach, Conference: American Control Conference, 17-19 June, Washington DC, 2025-2031.

8. Ren W., Beard R. W. (2004), Decentralized scheme for spacecraft formation flying via the virtual structure approach, Journal of Guidance, Control and Dynamics, 27(1), 73-82.

9. Reynolds, C.W. (1987), Flocks, herds and schools: a distributed behavioral model. In ACM SIGGRAPH Computer Graphics, Proceedings of ACM SIGGRAPH '87, Anaheim, USA, 27-31 July, ACM Press: New York, USA, 25-34.

10. Seo J., Ahn Ch., Kim Y. (2009), Controller Design for UAV Formation Flight Using Consensus based Decentralized Approach, AIAA Infotech@Aerospace Conference Unmanned, Unlimited Conference, 6-9 April, 248-259.

11. Shan J., Liu H.T. (2005), Close-formation flight control with motion synchronization, Journal of Guidance, Control and Dynamics, 28(6), 1316-1320.

12. Shao Z., Zhu X., Zhou Z., Wang Y. (2014), A Nonlinear Control of 2 D UAVs Formation Keeping via Virtual Structures, Intelligent Robotics and Applications, Lecture Notes in Computer Science, 8917, 420-431.

13. Virágh Cs., Vásárhelyi G., Tarcai N., Szörényi T., Somorjai G., Nepusz, T., Vicsek, T. (2014), Flocking algorithm for autonomous flying robots, Bioinspiration \& Biomimetics, 9(2), Article number 025012 (online).

14. Xingping Ch., Serrani A., Ozbay H. (2003), Control of leaderfollower formations of terrestrial UAVs, Proceedings. 42nd IEEE Conference on Decision and Control, 9-12 December, 498-503.

15. Yun B., Chen B.M., Lum K.Y., Lee T.H. (2008), A leader-follower formation flight control scheme for UAV helicopters, IEEE International Conference on Automation and Logistics, 1-3 Septemeber, $39-44$.

The research was realized within the project No S/WM/1/2017 and funded by Polish Ministry of Science and Higher Education. 